\title{
Multiple Ant Colony Optimization for Single Depot Multiple Trip Vehicle Routing Problems
}

\author{
Zhengmao Ye and Habib Mohamadian \\ College of Engineering, Southern University, Baton Rouge, LA 70813, USA \\ zhengmao_ye@subr.edu, habib_mohamadian@subr.edu
}

\begin{abstract}
:
The multiple trip vehicle routing problem involves several sequences of routes. Working shift of single vehicle can be scheduled in multiple trips. It is suitable for urban areas where the vehicle has very limited size and capacity over short travel distances. The size and capacity limit also requires the vehicle should be vacated frequently. As a result, the vehicle could be used in different trips as long as the total time or distance is not exceeded. Various approaches are developed to solve the vehicle routing problem (VRP). Except for the simplest cases, VRP is always a computationally complex issue in order to optimize the objective function in terms or both time and expense. Ant colony optimization (ACO) has been introduced to solve the vehicle routing problem. The multiple ant colony system is proposed to search for alternative trails between the source and destination so as to minimize (fuel consumption, distance, time) among numerous geographically scattered routes. The objective is to design adaptive routing so as to balance loads among congesting city networks and to be adaptable to connection failures. As the route number increases, each route becomes less densely packed. It can be viewed as the vehicle scheduling problem with capacity constraints. The proposed scheme is applied to typical cases of vehicle routing problems with a single depot and flexible trip numbers. Results show feasibility and effectiveness of the approach.

Keywords: Multiple Trip Vehicle Routing Problem, Multiple Ant Colony Optimization, Combinatorial Optimization
\end{abstract}

\section{Introduction}

One of the key problems in combinatorial optimization and integer programming is the well-known vehicle routing problem (VRP), where the optimal routes are subject to determination for a fleet of vehicles so that minimum total cost in terms of fuel consumption, labor, time, and distance can be reached with a set of constraints. To make a simple comparison, despite of the sophisticated lean burn engine design, modeling, control and optimization technologies, it is tough to improve fuel economy by $5 \%$. However, it is an easy task to save transportation efforts when vehicle routing optimization is applied. In fact, there is an ultimate goal of total expense minimization across many fields of transportation, distribution, exploration and exploitation. That is the reason why VRP is widely applied along with numerous optimal searching approaches [1-3]. The variant of the vehicle routing problem occurs with time windows and a limited number of vehicles. Tabu search is characterized by a holding list and mechanism to force dense packing within a route. Penalty for lateness is counted to relax time windows. The customer jobs can be inserted based on a hierarchical objective function that captures multiple objectives. The approach is effective from the stability points of view [4]. A hybrid variable 
neighborhood Tabu search is presented for the vehicle routing problem with multiple time windows. It proposes a minimum backward time slack algorithm which records the minimum waiting time and delay during route generation in order to adjust arrival and departure times backward. The implementation of the proposed heuristic method can compare with ant colony on benchmark instances involving multiple time windows [5]. A new Tabu search meta-heuristic is used for the time-dependent multi-zone multitrip vehicle routing problem with time windows. Tabu search yields high quality solutions and the proposed model clearly identifies major components of a decision set. The application for multiple neighborhoods improves both routing and vehicle assignment [6]. To minimize the number of trips, breaking ties is in favor of minimizing routing cost. An iterative solution approach decomposes the problem into simpler ones, each solved by heuristic study which are suitably combined to produce feasible solutions. Adaptive guidance mechanism is used to guide heuristics to improve current solution. Based on experiments for multi-regional scale distribution, the adaptive guidance mechanism is considerably efficient to reduce the overall number of vehicles within the limited computing time [7]. The multi-period vehicle routing problem with profit is also presented, whose goal is to determine routes for vehicles that maximize profitability from visited locations. An effective memetic algorithm is proposed with giant-tour representation. To efficiently evaluate the chromosome, a greedy procedure is developed to partition a given giant-tour into individual routes. The approach generates high quality solutions reasonably close to the best solutions, and significantly better than solutions obtained using heuristics by schedulers [8].

Artificial intelligence approaches can be applied to VRP as well. Genetic Algorithm (GA) is a global optimal approach to accentuate the high fitness on a basis of natural evolution theory which has applications in various fields. For instance, GA has been applied to biomedical sample Raman spectral characterization, such that fluorescence spectra and intrinsic spectra can be optimally separated [9]. When a variant of classical vehicle routing is needed where the vehicles are assigned to multiple routes within a period, the hybrid genetic algorithm with non-binary chromosome is proposed. This population-based heuristic is used to solve the VRP with multiple trips. It acts as the first attempt to use an evolutionary method rather than either Tabu search based or multi-level type heuristics. The GA generates a significant number of reasonable quality solutions in a short period when compared to other benchmarks. It has strong potential for future development in terms of the new chromosome generation and coding structure [10]. Another evolutionary optimization approach is Ant Colony Optimization (ACO), whose applications are also powerful and multidisciplinary. For example, it is proposed for edge and contour detection against false detection to extract distinctive information. The result outperforms Canny edge detection [11]. A new metaheuristic approach is inspired by foraging behaviors of real colonies of ants. It minimizes multiple hierarchical objective functions on the number of tours and total deadhead time. As a tradeoff between convergence and stagnation, improvement on route construction rule and pheromone updating rule are adopted. To deal with the fueling time constraint, a bipartite graphic model and an optimization algorithm are developed for set connection in a hub and spoke network system. It is more efficient and robust in problem solving [12]. The vehicle routing consists of optimally routing a fleet of vehicles with fixed capacity when travel is time dependent. The optimization method consists of finding solutions that minimize two hierarchical objectives of the tour times and total travel time. It is shown that when dealing with time constraints like hard delivery time windows, existing solutions are unfeasible and degree of unfeasibility increases along with variations of traffic conditions. The presented model is integrated with a robust shortest trail algorithm to compute time dependent trails between customers [13]. Parallel processing is presented to enhance performance of the ant colony algorithm. The performance of the multiple ant colony system algorithm applied to vehicle routing with time windows is investigated with respect to solution quality and computational effort. The study shows that multiple ACO outperforms sequential ACO. The weighting scheme improves performance in strategies that share pheromone information among all the ant colonies [14]. The multiple ant colony optimization (MACO) approach is applied to design a balanced and efficient supply chain network which maintains the balance between transit time and customers service. The 
focus is on effective allocation of customers to distribution centers with a tradeoff between the transit time and degree of imbalance of distribution centers. MACO technique is a modified form of ACO, where multiple colonies cooperate with each other for best customer allocation pattern. The proposed MACO shows better performance by means of both positive and negative feedback [15]. MACO is also developed to solve location routing problems with capacity constraints on depots and routes. It is decomposed into the facility location problem and multiple depot vehicle routing problem. The MACO algorithm applies a hierarchical ant colony structure to optimize location selection, customer assignment, and vehicle routing. Cooperation is made by exchanging information through pheromone updating between location selection and customer assignment. Computational results indicate competitiveness of MACO [16]. In this work, MACO will be employed to solve the multiple trip VRP optimization with single depot and capacity constraints.

\section{Multiple Ant Colony Optimization (MACO)}

MACO is generalized from classical ACO algorithms. In ACO, models of collective intelligence of the artificial ants are transformed into useful combinatorial optimization techniques. The colony of ants serve as cooperative agents who perform necessary tasks such as locating the shortest trail to the food source and sharing information with other ants by interacting between ants and environment and depositing a chemical substance called pheromone. With respect to the pheromone trails and heuristic information, probabilistic decisions are made by modeling colonies of ants as societies of moving agents to generate multiple routing preferences. The stochastic component allows artificial ants to explore multiple solutions of foraging trails through graphs. The artificial ants memorize positions and qualities of all existing trails such that better trail information is reached via communication. The use of heuristic information is helpful to direct the ants to promising search regions using the collective interaction among ants. Updated learning rules are determined by amount of pheromone accumulated. The learning process simulates the actual phenomenon in the natural world when ants are looking for food randomly between a colony and food sources. The pheromone trails to food sources being visited by some ants will have a larger chance to be followed by other ants. In turn, the pheromone amount will be reinforced if other ants also find food. The transition rule on a graph favors edges with more pheromone and less distance. The pheromone update rule deposits the amount of pheromone proportional to the length of the trail. The longer time ants commuting, the more time pheromones have to evaporate. Pheromone evaporates to avoid convergence to local optimization and excessive accumulation. Both reinforcing and evaporation processes will affect the pheromone density. Constraints should be applied to exploration and exploitation process. Via positive feedback, an optimal trail is accepted by all the ants at last. It represents a stigmergy system where ants exchange information mutually by depositing pheromones so that the single shortest trail to the source will be selected.

When multiple ant colonies coexist across the same area, single ACO algorithm should be extended to multiple ACO algorithm (MACO). Similarly, MACO is also inspired by the foraging behavior of groups of interconnected ant species. The population based metaheuristic optimization approach can be redesigned for each case of multiple ant colonies. The ants from several groups of colonies will work together by depositing pheromone on trials to communicate with each other, thus information could be shared more efficiently among multiple colonies. It results in a parallel structure by self-organizing multiple colonies at the same time. It is a stochastic process for the colonies to interact and communicate for cooperation, where ants from multiple colonies memorize good trails and deposit pheromone to show others by information exchange. Other ants at all colonies accept the pheromone information as a reference to promising regions. Hence, the exchange rule for colonies to collaborate with each other should be defined according to the mechanism of cooperation. The pheromone amount represents memory-based experiences of the ant colonies, so it is vital to choose a trail visibility function with 
maximal difference as potential candidates. The pheromone model parameters must be adjusted throughout the time, in order to increase pheromone amount with the correct choices and evaporate those with wrong ones. Once an ant from all colonies has completed trips, the pheromone on edges is updated. During the same cycle of iteration, the pheromone also evaporates on edges across the trail, making subsequent ants likely to select different edges to produce alternative solutions. The diversity is helpful to avoid local stagnation. Eventually the shortest trail is constructed as a combination of a set of selected routes. The best solutions will be marked by the ants from any individual colony. The MACO is formulated in four consecutive steps: initialization; edge selection; local pheromone update; global pheromone update via colony interaction. Iteration is continued until specified criterion is reached.

For the single depot MACO routing problem, the total number (M) of trips should be specified initially. The path visibility and pheromone trail capacity will be randomly generated to avoid initial stagnation. All locations to be visited are classified into M colonies, so that each colony covers a region with respect to the central depot. Based on initial assignment, the ants in each of $\mathrm{M}$ groups will conduct edge selection and local pheromone update procedures. Afterwards, M colonies will interact for global pheromone update. In the edge selection step, an individual ant moves from node $\mathrm{i}$ to node $\mathrm{j}$. The trail visibility function $\eta_{i, j}$ is defined as the reciprocal of the distance $D_{i, j}$ between two nodes $i$ and $j$. The selected edge is expected to have larger visibility. The selection rule for single ant is in (1).

$$
\mathrm{p}_{i, j}=\frac{\left(\tau_{i, j}\right)^{\alpha}\left(\eta_{i, j}\right)^{\beta}}{\sum\left(\tau_{i, j}\right)^{\alpha}\left(\eta_{i, j}\right)^{\beta}}
$$

where $\alpha$ is a parameter to adjust the impact of the amount of pheromone $\tau_{i, j} ; \beta$ is a parameter to adjust the impact of the heuristic visibility function $\eta_{i, j}$; $\tau_{i, j}$ is the pheromone amount on edge between $\mathrm{i}$ and $\mathrm{j} ; \eta_{\mathrm{i}, \mathrm{j}}$ is the trail visibility of between $\mathrm{i}$ and $\mathrm{j}$. Thresholding is applied to make a binary decision if a generated random number will be accepted or not. If a generated random number is below the threshold, then (1) does not apply. Instead $\mathrm{p}_{\mathrm{i}, \mathrm{j}}$ is assigned as one when the unvisited node $\mathrm{j}$ favors transition probability maximization, otherwise $\mathrm{p}_{\mathrm{i}, \mathrm{j}}$ is assigned as zero.

In the pheromone update step, local update is applied to any individual ant and global update is simply applied when ants in every colonies have completed tours. The former local update is independent of the colonies, while the latter global update contain information of colony interaction. The local pheromone update rule is defined as (2).

$\tau_{\mathrm{i}, \mathrm{j}}(\mathrm{t}+1)=(1-\rho) \tau_{\mathrm{i}, \mathrm{j}}(\mathrm{t})+\Delta \tau_{\mathrm{i}, \mathrm{j}}$

where $\rho$ is the rate of pheromone evaporation $(0<\rho<1) ; \tau_{i, j}$ is the pheromone amount on the edge between $\mathrm{i}$ and $\mathrm{j} ; \Delta \tau_{\mathrm{i}, \mathrm{j}}$ is the amount of pheromone deposited; $\Delta \tau_{\mathrm{i}, \mathrm{j}}=\eta_{\mathrm{i}, \mathrm{j}}$ when the ant travels on edge between $\mathrm{i}$ and $\mathrm{j}$. Pheromone evaporation is adopted to avoid the excessive accumulation of intensities. For the node not chosen by ants, the amount of pheromone decreases exponentially. To prevent searching procedures from stagnation, constraint minima of pheromone amount must be specified. The pheromone amount is simultaneously influenced by dual processes of evaporation and deposition.

The multiple colony interaction occurs at global update process. Define $\mathrm{F}^{\mathrm{K}}$ as the total cost of the heuristic solution for the colony $\mathrm{K}$ where $\mathrm{K}$ belongs to one of $\mathrm{M}$ different colonies in total. Also define $\mathrm{F}_{\mathrm{MAX}}$ and $\mathrm{F}_{\mathrm{MIN}}$ as maxima and minima of the objective functions among $\mathrm{M}$ colonies, then the global update process is defined as (3).

$\tau_{\mathrm{i}, \mathrm{j}}(\mathrm{t}+1)=(1-\rho) \tau_{\mathrm{i}, \mathrm{j}}(\mathrm{t})+\rho\left(\mathrm{R}^{\mathrm{K}} / \mathrm{F}^{\mathrm{K}}\right)$

where $\rho$ is the rate of pheromone evaporation and $\tau_{\mathrm{i}, \mathrm{j}}$ is the pheromone amount. $\mathrm{R}^{\mathrm{K}}$ is the ratio being defined as (4).

$\mathrm{R}^{\mathrm{K}}=\left(\mathrm{F}^{\mathrm{K}}-\mathrm{F}_{\mathrm{MIN}}\right) /\left(\mathrm{F}_{\mathrm{MAX}}-\mathrm{F}_{\mathrm{MIN}}\right)$

The local update process and global update process will both applied to the MACO problem in order to achieve a balance between exploration and exploitation. In this case, all the colony interactions are also taken into account so that global optimization for the multiple ACO system can be reached. It will be applied to multiple trip vehicle routing problem with single depot. 


\section{Multiple Trip Vehicle Routing Problem}

The multiple ant colony system has been applied to the multiple trip vehicle routing problem in this session. The objective function to be minimized is defined as (5), which covers the cost due to total distance being visited and penalty cost associated with all unvisited target nodes. It is also subject to the capacity constraint (6) and time constraint (7).

$$
\begin{array}{ll}
\mathrm{F}= & \sum_{\mathrm{j}=1}^{\mathrm{M}} \sum_{\mathrm{i}=0}^{\mathrm{N}+1}\left(\mathrm{D}_{\mathrm{ij}} \mathrm{Y}_{\mathrm{ij}}\right)+\mu \sum_{\mathrm{j}=1}^{\mathrm{M}} \sum_{\mathrm{i}=0}^{\mathrm{N}+1}\left(1-\mathrm{Y}_{\mathrm{ij}}\right) \\
\text { s.t. } & \sum_{\mathrm{i}=1}^{\mathrm{N}}\left(\mathrm{Y}_{\mathrm{ij}}\right) \leq C \quad \mathrm{j}=\{1,2, \ldots, \mathrm{M}\} \\
\text { s.t. } & \frac{\sum_{\mathrm{j}=1}^{\mathrm{M}} \sum_{\mathrm{i}=0}^{\mathrm{N}+1}\left(\mathrm{D}_{\mathrm{ij}}\right)}{\mathrm{mph}} \leq \mathrm{T}
\end{array}
$$

where $\mathrm{F}$ is the cost function, $\mathrm{M}$ is the total number of trips, $\mathrm{N}$ is the number of nodes to be visited at each trip, $\mathrm{D}_{\mathrm{ij}}$ is the distance (miles) between two nodes while $\mathrm{D}_{\mathrm{N}(\mathrm{N}+1)}=\mathrm{D}_{(0) \mathrm{N}}$ since both indices 0 and $\mathrm{N}$ refer to the central depot which serves as both the starting point and ending point, $Y_{i j}=\{0,1\}$ is a binary decision factor on if certain route has been selected or rejected, $\mu$ is a penalty factor defined to reduce the amount of unvisited nodes, $\mathrm{C}$ represents the capacity constraint using the maximal number of visited nodes on each trip (e.g. 5 or 10), mph (miles per hour) is the average local driving speed and $\mathrm{T}$ is the time constraint. For each trip (K: 1 to $\mathrm{M}$ ), $\mathrm{F}^{\mathrm{K}}$ is described as (8).

$$
F^{K}=\sum_{i=0}^{N+1}\left(D_{i j} Y_{i j}\right) \quad(j=K)
$$

All the colonies work together cooperatively. Via interaction among colonies, the MACO outperforms the ACO in complex optimization problems. Some assumptions for the current VRP problem are: A. All clients are served by exactly one vehicle per day locally; B. Single depot is assumed with the same starting and ending node; $\mathrm{C}$. The vehicle has fixed capacity, however, a vehicle with another capacity could be employed on separate date; D. The vehicle may drive arbitrary number of routes as long as the total driving time won't be exceeded. Eighty locations are randomly generated where the single depot is selected near the center of the graph (Figure 1). Without loss of generality, the number of trips has been selected as 1, 2, 3, 5, 8, and 10, respectively. Iteration upper bound is selected to be 10,000 . Then the cost function and corresponding iteration number are calculated using the proposed MACO algorithm. Simulation results for different cases are plotted in Figures. 2-13, respectively, representing diverse number of trips. 


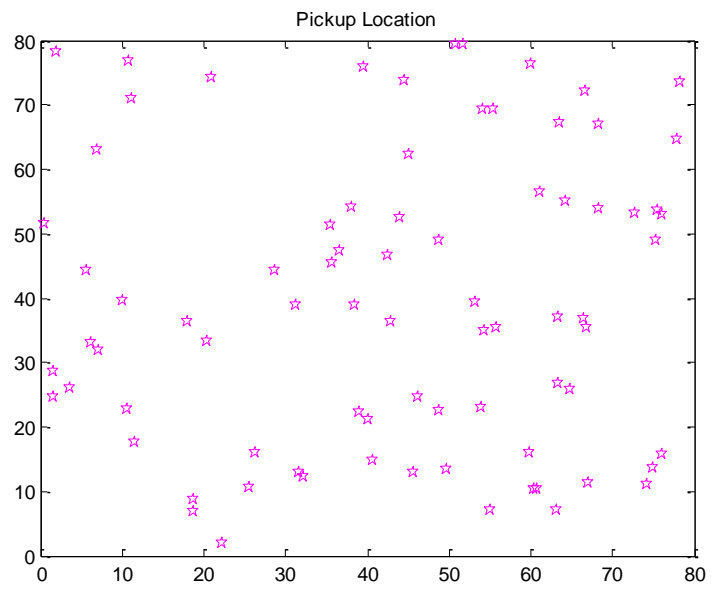

Figure 1: Locations of Multiple Trip VRP with Single Depot

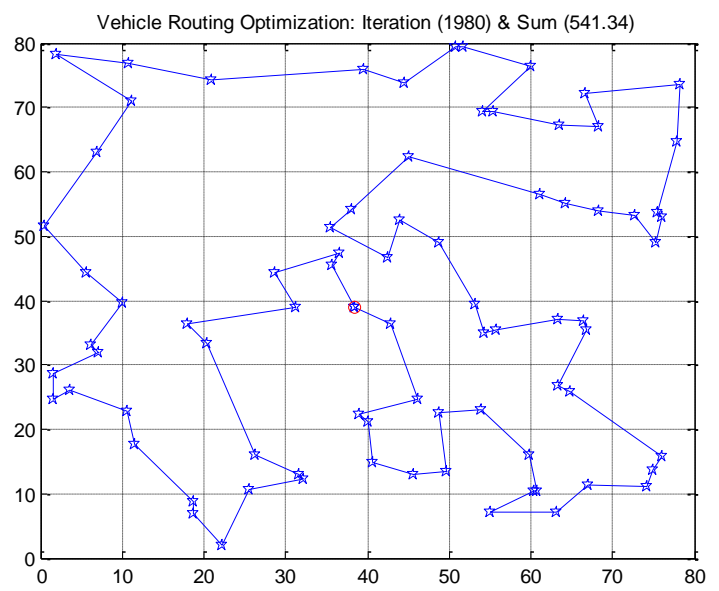

Figure 2: Routing Graph for 1-Trip VRP

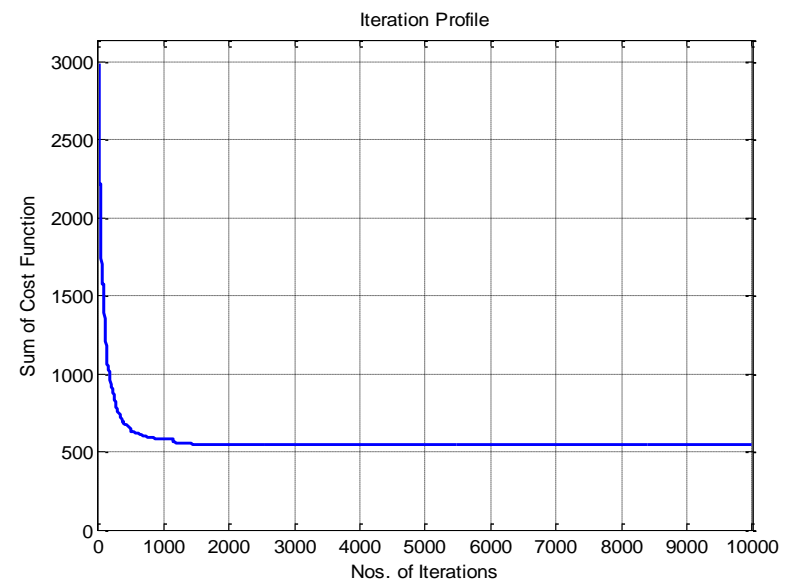

Figure 3: MACO Iteration for 1-Trip VRP 


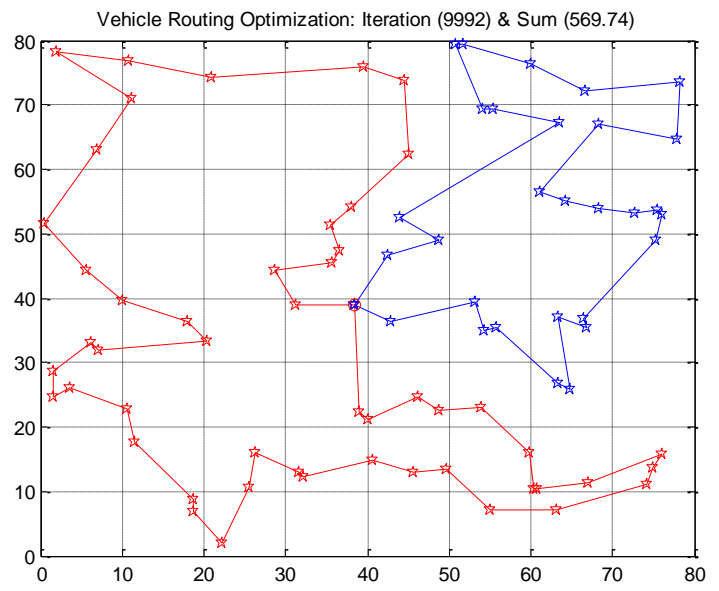

Figure 4: Routing Graph for 2-Trip VRP

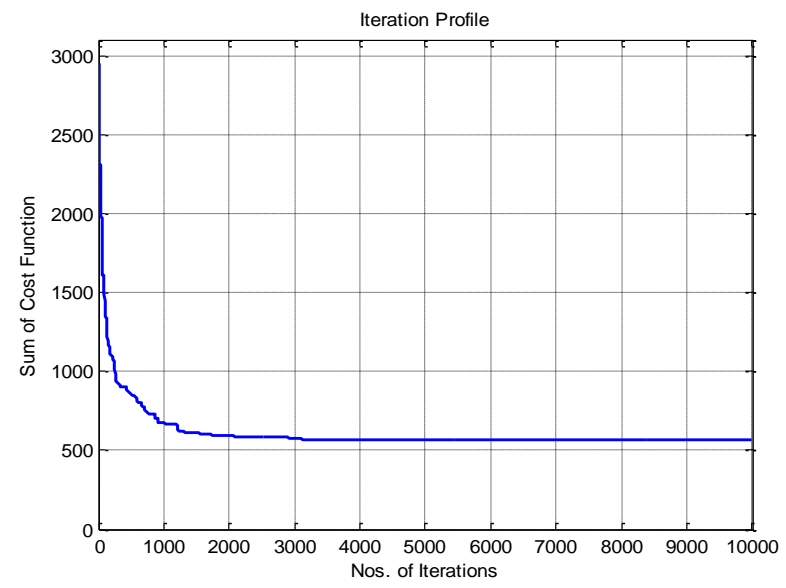

Figure 5: MACO Iteration for 2-Trip VRP

Vehicle Routing Optimization: Iteration (9185) \& Sum (636.51)

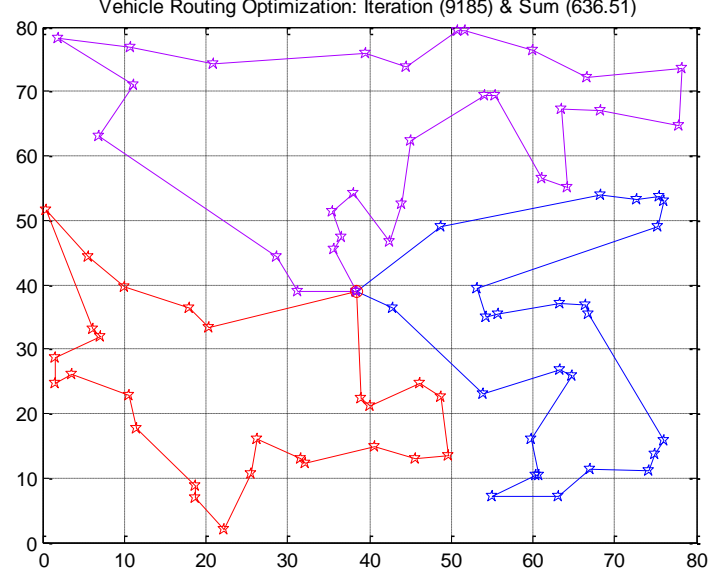

Figure 6: Routing Graph for 3-Trip VRP 


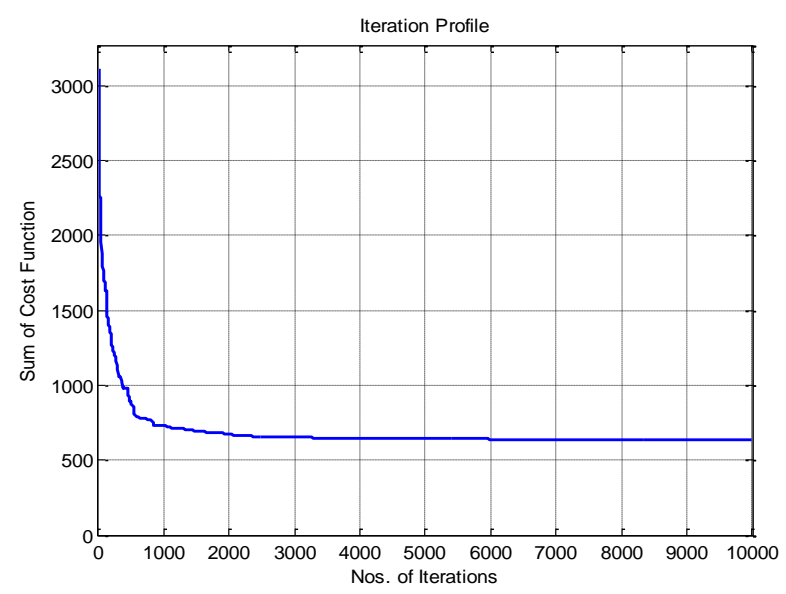

Figure 7: MACO Iteration for 3-Trip VRP

Vehicle Routing Optimization: Iteration (9821) \& Sum (743.82)

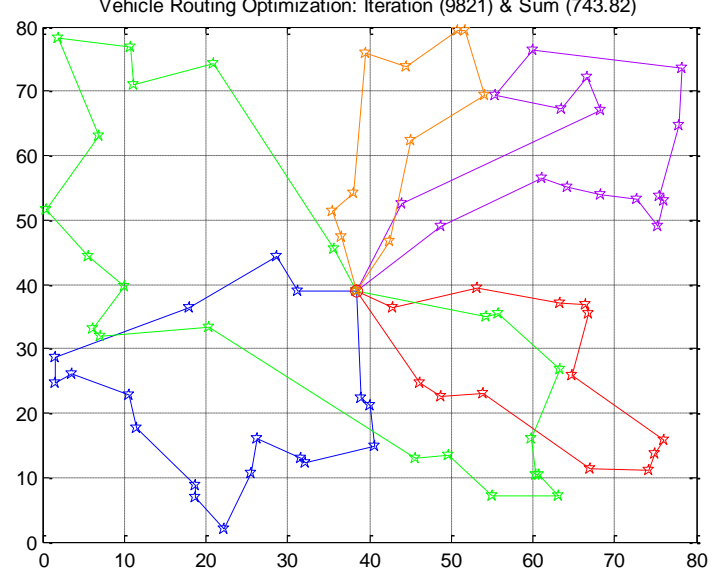

Figure 8: Routing Graph for 5-Trip VRP

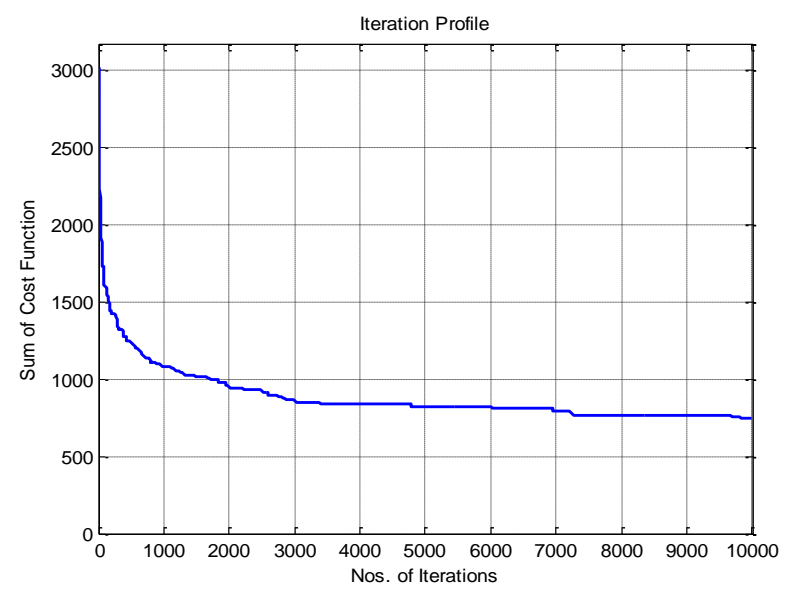

Figure 9: MACO Iteration for 5-Trip VRP 


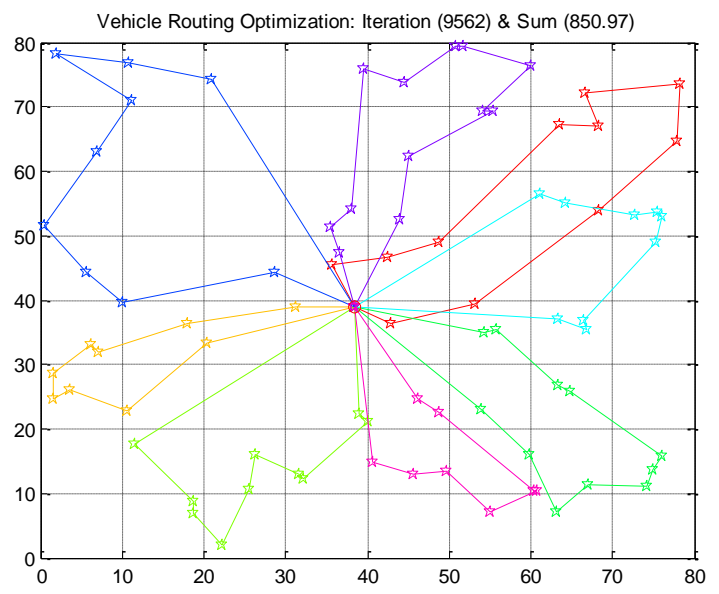

Figure 10: Routing Graph for 8-Trip VRP

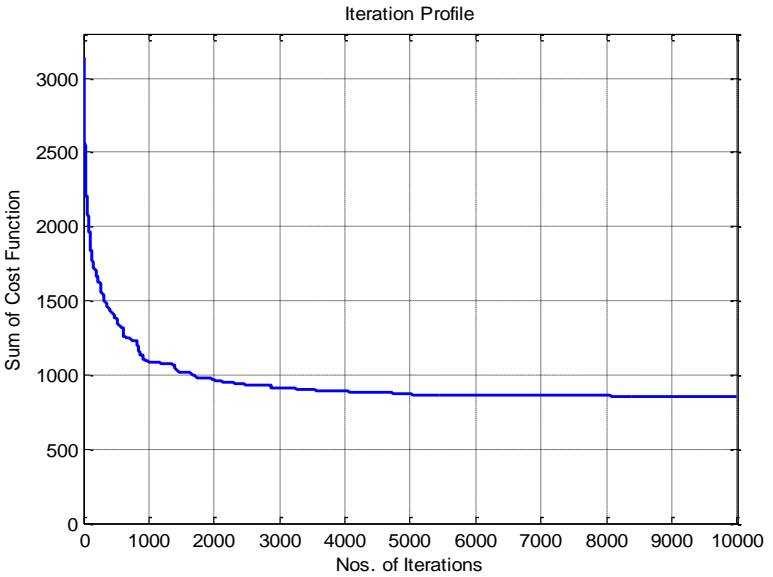

Figure 11: MACO Iteration for 8-Trip VRP

Vehicle Routing Optimization: Iteration (9726) \& Sum (989.64)

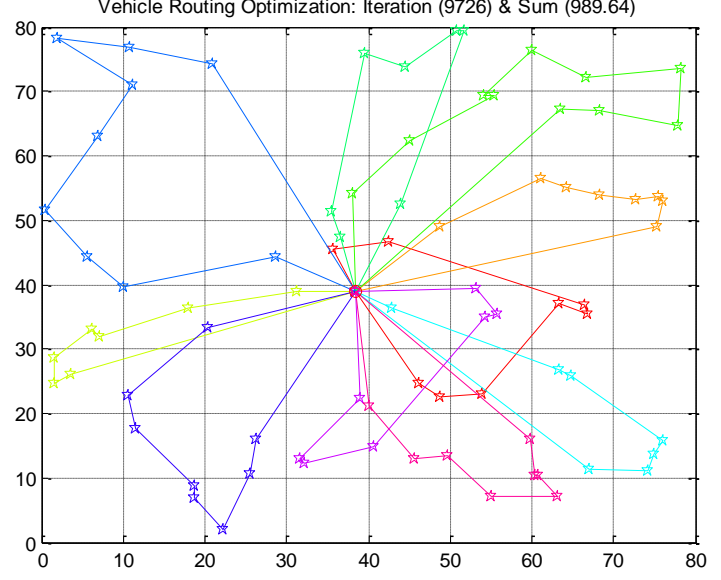

Figure 12: Routing Graph for 10-Trip VRP 


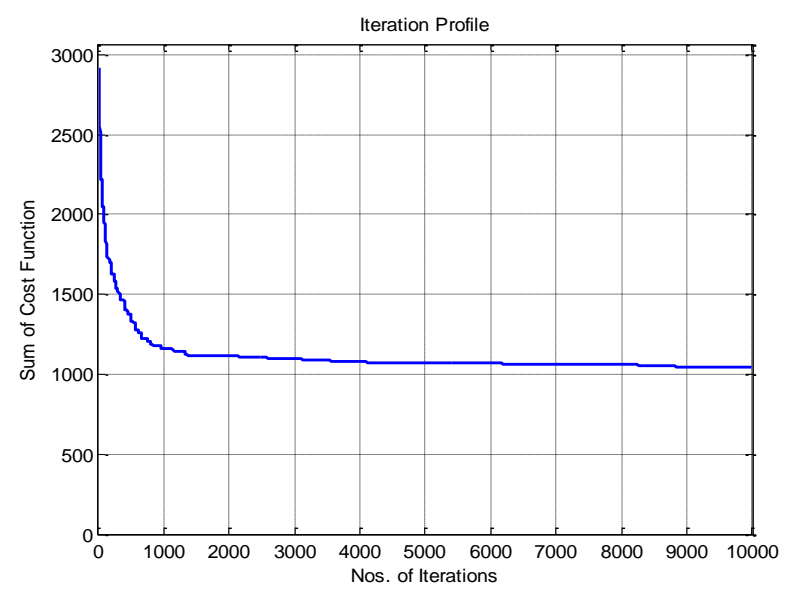

Figure 13: MACO Iteration for 10-Trip VRP

\section{Comparison of Multiple Trip VRP Cases}

In last session, numerical simulations on VRP with single depot using multiple ant colony optimization are conducted. Multiple colonies of ants are essential to locate the shortest trail to make multiple routing preferences for any number of trips. Typical numbers $(1,2,3,5,8,10)$ of routes are assumed when the upper bound of iteration is set as 10,000. The minimal total cost and its corresponding iteration number for multiple cases is shown in Table 1. The convergence rate of the single trip VRP optimization is significantly faster than the multiple trip VRP optimization. The minimal total cost is smallest for single route VRP, which keeps monotonically increasing along with the increment of the trip number when single depot is assumed. The problem with vehicle capacity constraints (maximal number of nodes in each trip) is applied so that multiple trip vehicle routing is scheduled. For each case, successful heuristic solution is achieved using MACO optimization.

\begin{tabular}{|c|c|c|c|c|c|c|}
\hline $\begin{array}{c}\text { No. of } \\
\text { Trips }\end{array}$ & 1 & 2 & 3 & 5 & 8 & 10 \\
\hline Minimal Cost & $\mathbf{5 4 1 . 3 4}$ & $\mathbf{5 6 9 . 7 4}$ & $\mathbf{6 3 6 . 5 1}$ & $\mathbf{7 4 3 . 8 2}$ & $\mathbf{8 5 0 . 9 7}$ & $\mathbf{9 8 9 . 6 4}$ \\
\hline $\begin{array}{c}\text { No. of } \\
\text { Iterations }\end{array}$ & $\mathbf{1 9 8 0}$ & 9992 & 9185 & 9821 & 9562 & 9726 \\
\hline
\end{tabular}

Table 1: Minimal Total Cost for Single Depot Multiple Trip VRP 


\section{Conclusions}

The multiple trip vehicle routing problem with the capacity constraint is stated to manage a set of routes and serve a number of locations, with maximal profitability and minimal cost (fuel consumption, time, distance). The single depot VRP is selected and then solved by multiple ant colony optimization. The novel MACO algorithm is designed and applied to both the single trip vehicle routing and multiple trip vehicle routing problems. When an upper bound of iterations is set, numerical simulations are conducted to evaluate feasibility of the proposed approach in dealing with the number of trips using single vehicle with fixed capacity. Routing graphs for diverse number of trips have been plotted where the minimum cost function is reached within a specified iteration maximal number. The iteration profile of the cost function versus iteration number is also provided. The effectiveness of the MACO algorithm on each case has been observed. Comparison is also made on a basis of the relationship among routing graphs, numbers of trips and total costs after certain iterations.

\section{References}

[1] A. Engelbrecht, "Computational Intelligence: An Introduction", 2nd Edition, ISBN: 978-0-47003561-0, November 2007, Wiley Press

[2] F. Lewis, D. Vrabie, V. Syrmos, "Optimal Control", ISBN: 978-0-470-63349-6, February 2012, Wiley Press

[3] Z. Ye and Z. Li, "Impact of Lean-Burn Control Technology on the Fuel Economy and NOx Emission of Gasoline Engines", ISSN: 0954-4070, pp. 1041-1058, Vol. 224, No. 8, Proceedings of the Institution of Mechanical Engineers, Part D, Journal of Automobile Engineering, 2010

[4] H. Lau a, M. Sim, K. Teo, "Vehicle Routing Problem with Time Windows and A Limited Number of Vehicles", European Journal of Operational Research 148 (2003) 559-569

[5] S. Belhaiza, P. Hansen, and G. Laporte, "A Hybrid Variable Neighborhood Tabu Search Heuristic for the Vehicle Routing Problem with Multiple Time Windows", Computers and Operations Research, August, 2013

[6] P. Nguyen, T. Crainic, and M. Toulouse, "A Tabu Search for Time-dependent Multi-zone Multitrip Vehicle Routing Problem with Time Windows", EU Journal of Operational Research 231 (2013) 43-56

[7] M. Battarraa, M. Monacib, and D. Vigoa, "An Adaptive Guidance Approach for the Heuristic Solution of a Minimum Multiple Trip Vehicle Routing Problem", Computers \& Operations Research 36 (2009) $3041-3050$

[8] Z. Zhang, O. Che, B. Cheang, A. Lim, and H. Qin, "A Memetic Algorithm for Multiperiod Vehicle Routing Problem with Profit”, European Journal of Operational Research 229 (2013) 573-584

[9] Z. Ye, "Artificial Intelligence Approach for Biomedical Sample Characterization Using Raman Spectroscopy", IEEE Transactions on Automation Science and Engineering, Vol. 2, No. 1, pp. 67 73, January, 2005

[10] S. Salhi, R. Petch, "A GA Based Heuristic for the Vehicle Routing Problem with Multiple Trips", Journal of Mathematical Modelling and Algorithms (2007) 6:591-613

[11] Z. Ye, H. Mohamadian, "Strengthen Accuracy of Feature Recognition via Integration of Ant Colony Detection and Adaptive Contour Tracking", Proceedings of the 2011 IEEE Congress on Evolutionary Computation, pp. 1799-1804, June 5-8, 2011, New Orleans, USA

[12] H. Wang, J. Shen, "Heuristic Approaches for Solving Transit Vehicle Scheduling Problem with Route and Fueling Time Constraints", Applied Mathematics and Computation 190 (2007) 12371249 
[13] R. Montemanni, N. Casagrande, A. Rizzoli, L. Gambardella, "Time Dependent Vehicle Routing Problem with a Multi Ant Colony System", European Journal of Operational Research, Volume 185, Issue 3, 16 March 2008, Pages 1174-1191

[14] I. Ellabib, P. Calamai a, O. Basir, "Exchange Strategies for Multiple Ant Colony System", Information Sciences 177 (2007) 1248-1264

[15] R. Montemanni, N. Casagrande, A. Rizzoli, L. Gambardella, "Time Dependent Vehicle Routing Problem with a Multi Ant Colony System", European Journal of Operational Research, Volume 185, Issue 3, 16 March 2008, Pages 1174-1191

[16] I. Ellabib, P. Calamai a, O. Basir, "Exchange Strategies for Multiple Ant Colony System", Information Sciences 177 (2007) 1248-1264 